\title{
Double Slit Experiment
}

\section{Paul T E Cusack*}

BScE, DULE, 23 Park Ave. Saint John, NB E2J 1R2, Canada

Corresponding author: Paul T E Cusack, BScE, DULE, 23 Park Ave. Saint John, NB E2J 1R2, Canada

\begin{tabular}{|c|c|}
\hline ARTICLE INFO & ABSTRACT \\
\hline Received: 㹸 October 13, 2020 & Citation: Paul T E Cusack. Double Slit Experiment. Biomed J Sci \& Tech Res 31(3)-2020. \\
\hline Published: 慧 October 29, 2020 & BJSTR. MS.ID.005109. \\
\hline
\end{tabular}

\section{Introduction}

In this paper, we consider the famous double slit experience in light of AT Math. We see that familiar values for certain variables

reoccur. This model can be used on problems such as virus transmission. We begin with the familiar sine equals cosine (Figure 1).

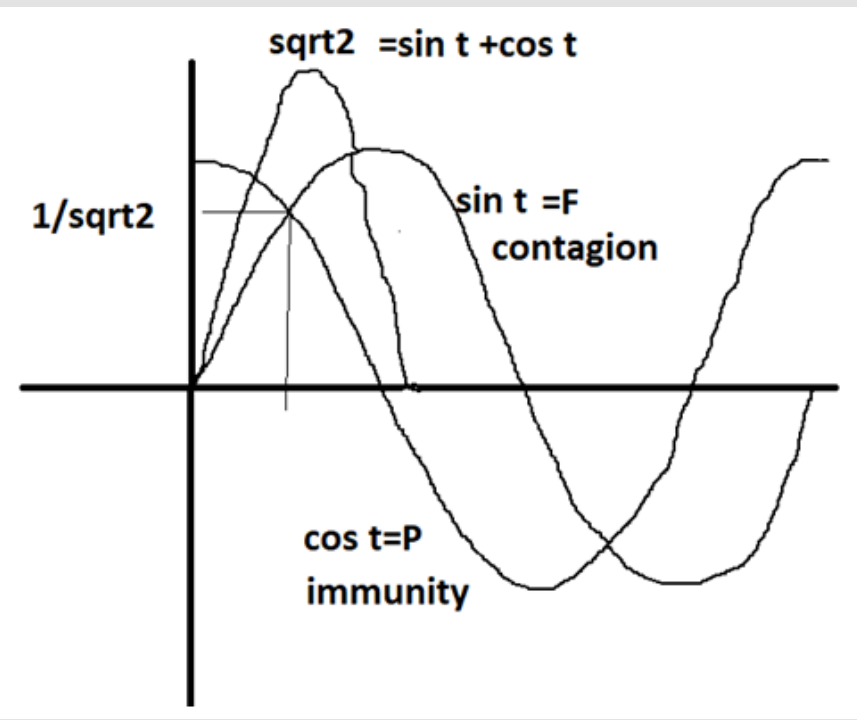

Figure 1: Immunity and Contagion.

Sine $=$ cosine
Immunity $=$ Cont
$\cos \theta=\sin \theta$
$\mathrm{Mv}=1 / \mathrm{t}$
$\operatorname{Ln} \mathrm{t}(1 / \sqrt{2})=1 / \mathrm{t}$
$(1 / \sqrt{2}) \operatorname{Ln} \mathrm{t}=1 / \mathrm{t}$
$(1 / \sqrt{2}) \mathrm{y}=\mathrm{y}^{\prime}$

$$
\begin{aligned}
& \sqrt{2} \mathrm{y}=y^{\prime} \\
& \int \sqrt{2} \mathrm{y}=\int y \\
& (\sqrt{2}) \mathrm{y}^{2} / 2=y \\
& 1 / \sqrt{2} \times \mathrm{y}=1 \\
& \mathrm{y}=\sqrt{2} \\
& \sin \theta+\cos \theta \\
& =\sin 45+\cos 45=
\end{aligned}
$$



$=(1 / \sqrt{2})+(1 / \sqrt{2})$
$\mathbf{E}=\sqrt{ } \mathbf{2}=\max$ of $\sin +\cos$
(Figure 2)
$=2 / \sqrt{2}$
$E=h v$

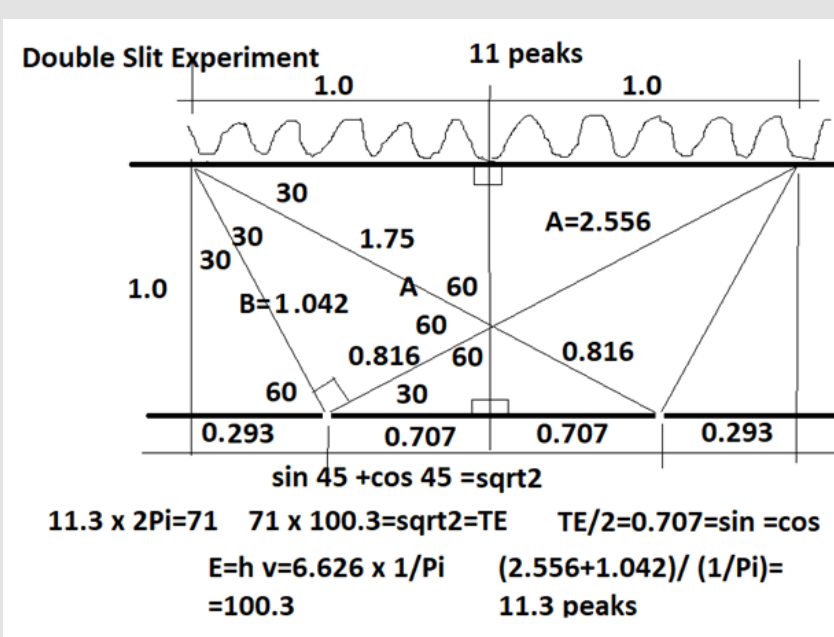

Figure 2: Double Slit Experiment.

$$
\begin{aligned}
& =6.626 \times 1 / \pi \\
& =208.82 \\
& y=f(x)=1 / \sigma \sqrt{ }(2 \pi) \exp \left[(-1 / 2(X-\mu) / \sigma]^{2}\right. \\
& \exp \left[(-1 / 2(X-\mu) / \sigma]^{2}=\exp [-1 / 2(0) / \sigma]^{2}=\exp 0=1\right. \\
& \mathrm{y}=\mathrm{f}(\mathrm{x})=1 / \sigma \mathrm{V}(2 \pi)[1] \\
& \mathrm{E}=\mathrm{y}=2.08816(\sqrt{ }(2 \pi)=1 / \sigma \\
& \sigma=.191
\end{aligned}
$$

\section{Entropy}

$$
\begin{aligned}
& S=1 / 2\left(\operatorname{Ln}\left[2 \pi e \sigma^{2}\right]\right. \\
& =-0.2366 \\
& \mathrm{t}^{2}-\mathrm{t}-1=\mathrm{S}=-0.2366 \\
& \mathrm{t}^{2}-\mathrm{t}-1.2366=0 \\
& \mathrm{t}=1.719 ; 0.719
\end{aligned}
$$$$
E=0.582 ; 1.39
$$

$\mathrm{M}=\mathrm{Ln} \mathrm{t}$$$
=\operatorname{Ln} 1.719
$$$$
=542
$$$$
\mathrm{M}=\operatorname{Ln} 0.719
$$$$
=330
$$$$
T E=M[0.15915]=542(1 / 2 \pi)=0.863 \sim 1 / \sin 60^{\circ}
$$

$$
\begin{aligned}
& \mathrm{TE}=330[1 / 2 \pi]=52.52 \\
& -1 / 2[\mathrm{X}-\mu) / \sigma]^{2}=1 \\
& {[(\mathrm{X}-\mu)(0.191)]^{2}=-2} \\
& (\mathrm{X}-\mu)^{2}=-0.7296 \\
& (\mathrm{X}-1 / 2)^{2}=-0.73 \\
& \mathrm{X}^{2}-\mathrm{X}+1 / 4=-0.73 \\
& \mathrm{X}^{2}-\mathrm{X}-1=0
\end{aligned}
$$

\section{GMP}

$$
\begin{aligned}
& \mathrm{V}=\mathrm{iR} \\
& 105 \mathrm{i}(1) \\
& \mathrm{i}=\mathrm{t}^{2} \\
& \sqrt{105}=\mathrm{t} \\
& \mathrm{t}=10.247 \\
& \mathrm{X}^{2}-\mathrm{X}-1=0 \\
& 10247^{2}-10247-1=9397 \sim 940 \\
& f(x)=1 /(0.191) \sqrt{ }(2 \pi) \exp [(940-0.5) / 0.191]^{2} \\
& =[1 / 0.191 \sqrt{2}] \times(201.7) \\
& =0.7467 \sim 3 / 4=1 / \mathrm{s} \\
& \mathrm{s}=\mathrm{t} \\
& \mathrm{E}=3 / 4=1 / \mathrm{s}=1 / \mathrm{t}(\text { Figure } 3) \\
& \Delta L=d \sin \theta
\end{aligned}
$$




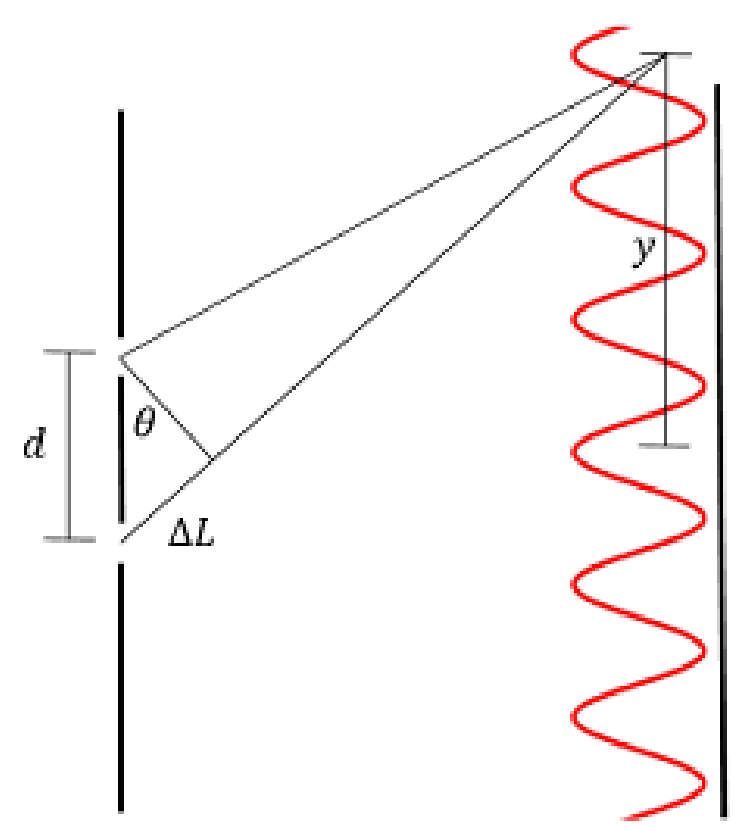

Figure 3: Double Split Experiment.

Note: https://brilliant.org/wiki/double-slit-experiment/

$$
\begin{aligned}
& \Delta \mathrm{L}=(1.042-2.556)=1.514 \\
& 1.514=\sqrt{2} \sin \theta \\
& \sin \theta=-1.514 / \sqrt{2} \\
& =-1.07056 \\
& \theta=-0.618 \\
& d \sin \theta=n \lambda \\
& (\sqrt{2})(-.107056)=11.3 \lambda \\
& \lambda=1.339=1 / E=t \\
& t^{2}-t-1=E \\
& E=546 \\
& G M P \quad E=-1.247 \\
& d \theta=y
\end{aligned}
$$

\section{ISSN: 2574-1241}

DOI: 10.26717/BJSTR.2020.31.005109

Paul T E Cusack. Biomed J Sci \& Tech Res

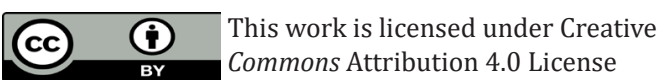

Submission Link: https://biomedres.us/submit-manuscript.php

$$
\begin{aligned}
& \mathrm{y}=\theta=-.0 .618 \\
& \mathrm{n} \lambda=\mathrm{dy} / \mathrm{D} \\
& 11.3 \lambda=(1 / \sqrt{2})(-1.0786) /(1) \\
& \lambda=1.047 \sim 105=\mathrm{V}+ \\
& \lambda=\mathrm{h} / \overline{\mathrm{P}} \\
& 104.7=6.626 / \overline{\mathrm{P}} \\
& \overline{\mathrm{P}}=158 . \sim \pi / 2=\cos \theta \\
& \theta=809=1 / \mathrm{c}^{4} \\
& \mathrm{y}=\mathrm{n} \lambda \mathrm{D} / \mathrm{d} \\
& 1=11.3(\lambda)(1) /(\sqrt{2}) \\
& \lambda=8 \\
& 1 / \lambda=1.25=\mathrm{Emin}
\end{aligned}
$$

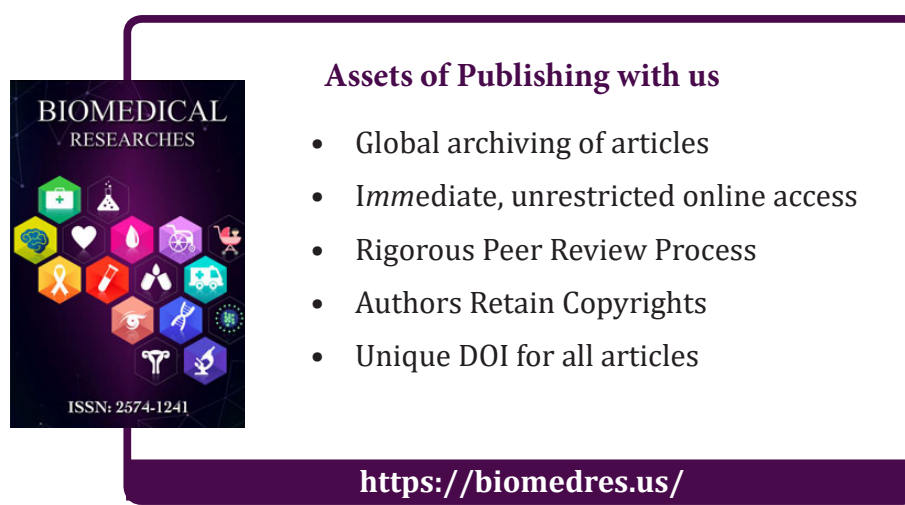

